\title{
A Preliminary Diffusion Tensor Imaging Study: Analysis of Whole Brain according to the Affected Side in Tinnitus
}

\author{
Shrestha $\mathbf{L}^{1,2}$ \\ ${ }^{1}$ Patan Academy of Health Sciences, Lagankhel, Lalitpur, Nepal. \\ ${ }^{2}$ Huazhong University of Science and Technology, Tongji Medical College, Wuhan, China. \\ Correspondence to: Dr Lochan Shrestha; Email: lochanshrestha@pahs.edu.np
}

\begin{abstract}
Aim: To investigate the role of white matter integrity in the pathophysiology of tinnitus, and also to analyze the whole brain for white matter changes quantitatively by comparing tinnitus patients with healthy controls based on the affected side of the tinnitus using Tract Based Spatial Statistics (TBSS). Methods: Total of 41 right-handed tinnitus patients and 35 agematched right-handed healthy controls were initially examined non-invasively in resting-state using the 3.0T MRI scanner. We obtained the estimated Fractional anisotropy (FA), mean diffusivity (MD), Axial Diffusivity (AD), Radial Diffusivity (RD), and Mode of Anisotropy (MO) data for each subject. TBSS was used to perform group statistical analysis of DTI data from each group. Results: Compared to controls in right-sided tinnitus patients, FA and MO were decreased, and $\mathrm{MD}$ was normal with decreased $\mathrm{AD}$ and increased RD. FA and $\mathrm{AD}$ showed similar decreased values $\left(t>1.5, \mathrm{FWE}_{\text {corrected }} \mathrm{P}<0.05\right)$ in the right corticospinal tract, right superior longitudinal fasciculus, and right inferior fronto-occipital fasciculus. Compared to controls with left-sided tinnitus patients, FA and MO were decreased, and MD was also increased with decreased $\mathrm{AD}$ with increased $\mathrm{RD}$ in patients. $\mathrm{MO}$ showed significantly decreased values $\left(\mathrm{t}>1.5, \mathrm{FWE}_{\text {corrected }} \mathrm{P}<0.05\right)$ of $\mathrm{MO}$ in Forceps major, bilateral cingulum (especially the left), and bilateral anterior thalamic radiation. Bilaterally affected tinnitus patients showed no difference when compared to controls. Conclusion: After analyzing the whole brain for white matter changes quantitatively using TBSS, changes were observed in unilaterally affected tinnitus patients compared to controls. Our findings indicated crossmodal plasticity could have caused changes in somatosensory, audio-visual, limbic, and attention pathways.
\end{abstract}

Keywords: Tinnitus; DTI; TBSS; Asymmetry; Primary Auditory Cortex. 


\section{INTRODUCTION}

Tinnitus is a phantom perception of sound without the presence of external auditory stimuli. Advances have been made for its management based on research from various studies. They focus on documenting functional and structural changes in tinnitus patients to explain the possible pathophysiological mechanisms involved. Jastreboff ${ }^{1}$ proposed tinnitusretraining therapy based on this model and has gained popularity. However, their role in cognitive behavioral therapies is still being studied ${ }^{2},{ }^{3}$ and a model, which accounts for changes according to affected side, has not been considered. Therefore, their management still remains a challenge.

Yoo et al. ${ }^{4}$ presented the first Diffusion tensor imaging (DTI) study as a preliminary report after examining 10 tinnitus patients without controls to quantify white matter changes in these patients but lacked generalizability. Lee et al. ${ }^{5}$ investigated parietal and frontal cortices and observed reduced white matter integrity in association fibers connecting these two cortices in their patients compared to their control group, which was correlated with distress and cognitive deficits. Later in 2010, Crippa et al. ${ }^{6}$ used a probabilistic tractography method to track classical auditory pathway and demonstrated increased fractional anisotropy (FA) connecting auditory cortex and the amygdala in patients compared with their controls. In 2011, Husan et al. ${ }^{7}$ combined DTI with tractbased spatial statistics (TBSS) to observe white matter changes and Voxel based morphometry (VBM) to demonstrate structural gray matter changes of the whole brain in tinnitus patients to disassociate tinnitus and hearing loss from hearing loss alone. This DTI study also uses TBSS for the analysis of the whole brain to demonstrate white matter changes in various non-auditory and auditory parts of the brain. However, no study has considered the effect of tinnitus based on the side of tinnitus perception i.e. either unilaterally or bilateral, and left or right.

The aim of this case-control study was to investigate the role of white matter integrity in the pathophysiology of tinnitus. The objectives of the study were to analyze the whole brain for white matter changes quantitatively by comparing tinnitus patients with healthy controls based on the affected side of the tinnitus using Tract Based Spatial Statistics (TBSS). Therefore, this study investigated tinnitus related neuronal dysfunction and plasticity, which led to white matter integrity changes and whether they differed according to the affected side.

\section{MATERIALS AND METHODS}

\section{Subjects}

The experiment was conducted in agreement with the guidelines of the Declaration of Helsinki and was approved by the Ethics Committee of Tongji Medical College of Huazhong University of Science and Technology, China. Seventy-six subjects were recruited with informed and written consent. The participants were divided into groups of patients according to the side of perceived tinnitus and aged-matched healthy volunteers as controls. Total of 41 tinnitus patients (11 left-side, 15 right-sided, and 
15 bilateral) and 35 age-matched righthanded healthy controls were initially examined non-invasively in resting-state using the 3.0T MRI scanner. All of the acquired data were carefully examined for artifacts.

All the participants were right handed, and had no known history of congenital or acquired neurological or psychiatric disease. The patients enrolled from the Otorhinolaryangeology (ENT) Outpatient Department in Union Hospital at Wuhan, China and were of Chinese Han descent from September to November 2013. All data were manually examined for any artifacts and 4 participants were excluded. Therefore, we had a total of 72 remaining participants.

Table 1 tabulates the subject characteristics and paired T-test demonstrating age match of patients and their controls.

Table 1: Characteristics of included participants.

\begin{tabular}{|l|c|c|c|c|c|}
\hline & Left & Right & Bilateral & Control & Total \\
\hline No. of Subjects & 10 & 14 & 14 & 34 & 71 \\
\hline Mean Age \pm SD & $38.1 \pm 19$ & $45.9 \pm 17$ & $46.9 \pm 15.3$ & $46.5 \pm 16.2$ & $44.35 \pm 15.6$ \\
\hline Male/Female & $5 / 5$ & $8 / 6$ & $9 / 5$ & $17 / 17$ & $39 / 33$ \\
\hline P-value & 0.98 & 0.86 & 0.84 & - & - \\
\hline
\end{tabular}

P-Value was obtained after paired T-test between age of controls and groups.

\section{Data Acquisition}

All of the tests were non-invasive, and DTI data were obtained from a 3.0 Tesla Magnetic Resonance Scanner (Siemens TrioTim) using an eight-channel phased array head coil and a single-shot EPI sequence with Time of Repetition (TR) = $4600 \mathrm{~ms}$, Time of Echo $(\mathrm{TE})=175 \mathrm{~ms}$, and flip angle $=90^{\circ} .126$ directions of diffusion were encoded with b-values from 0 to $7000 \mathrm{~s} / \mathrm{mm}^{2}$, Field of View $($ FOV $)=$ $256 \times 256 \mathrm{~mm}$, acquisition matrix = $128 \times 128$, slice thickness $=4 \mathrm{~mm}$, slice space $=1 \mathrm{~mm}$, and voxel-dimension $=$ $2 \times 2 \times 5 \mathrm{~mm}$. The total average scan time was about 10 minutes for each participant.

\section{Data Preparation}

MRIcro was used to convert the raw data into Neuroimaging Informatics
Technology Initiative (NIfTI) format for FSL toolbox (FMRIB Software, http://fsl.fmrib.ox.ac.uk/fsl/fslwiki/). We used Brain Extraction toolbox (BET) to remove any non-brain tissue ${ }^{8}$ and Diffusion Toolbox (FDT) to correct all data for eddy current and head motion and for local fitting of diffusion tensors to calculate the diffusion index ${ }^{9}$ We therefore obtained the estimated FA, MD, $\mathrm{AD}, \mathrm{RD}$, and $\mathrm{MO}$ data for each subject.

\section{Data Analysis}

Each individual patient along with their age-matched control was classified into their group respective of the side of perceived tinnitus. Therefore, all the patients and their age-matched controls were grouped into left-sided tinnitus group, bilateral tinnitus group, and rightsided tinnitus group for analysis. Tract 
based spatial statistics (TBSS) was used to perform group statistical analysis of DTI data from each group.

\section{Analysis of FA data}

We used the FMRIB58_FA standard-space image as the target for a voxel-wise nonlinear registration of multiple subject FA images. The transformed images were then brought to $1 \times 1 \times 1 \mathrm{~mm}$ MNI152 space and averaged to create a mean FA image. We threshold the mean FA value at 0.2 to remove voxels representing primarily grey matter or CSF in the majority of subjects. It also meant that the skeleton did not run right up to the outermost edges of the cortex reducing inter-subject variability. Using the mean threshold value, a mean FA skeleton was then projected in all-inone 4D FA images, which represented all the aligned FA data of all subjects in a group. A line represented the center of each white matter tract. The data could now be used for voxel-wise statistics. We designed a simple univariate linear model, i.e., process each skeleton voxel independently, applying the general linear model (GLM). Therefore, we had contrasts of Control > Patients and Controls < Patients for comparisons. We used a Monte Carlo permutation-based approach against null distribution using Randomize tool from FSL. We selected the default (5000) number of permutations for Family Wise Error (FWE) correction to search for significant data in the skeleton by thresholding cluster extent for multiple comparisons. Since thresholding is arbitrary, we arbitrarily selected cluster extent threshold levels. We followed the above steps for all 3 groups containing the patients and their age- matched controls.

\section{Analysis of non-FA data}

The non-linear warps and skeleton projection for FA maps could also be applied to non-FA data to create non-FA maps for statistical analyses. The original non-linear registration, which was applied to FA, was also applied to the non-FA data. They were then merged into a 4D file and to form a mean non-FA image. They were then projected into a group original mean FA skeleton to produce a 4D non-FA skeleton as well as a mean non-FA skeleton. We then used the skeletonized data for statistical analysis using the same GLM design and Randomize tool from FSL based on Monte Carlo Permutation approach. We selected the default (5000) number of permutations for FWE correction to search for significant data in the skeleton by thresholding cluster extent for multiple comparisons. Since thresholding is arbitrary, we selected cluster extent thresholding of 1.5 and 3 . We performed the above steps to each non-FA data of each group, which included MD, MO, AD, and RD.

\section{Anatomical White Matter Labeling}

We used the atlasqueryand cluster commands, part of the FSL toolkit, to localize the voxels based on John Hopkins University's (JHU)White matter tractography atlas and ICBM-DTI-81 White-Matter Labels. The process and generation of white matter labels were automated using a script (http://brainder.org/2012/07/30/automaticatlas-queries-in-fsl/). 


\section{RESULTS}

We labeled the DTI data based on JHU White Matter Tractography Atlas. FA and $\mathrm{AD}$ of the right-sided tinnitus patients, compared to controls, showed similar decreased values $\left(\mathrm{t}>1.5, \quad \mathrm{FWE}_{\text {corrected }}\right.$ $\mathrm{P}<0.05)$ in the right corticospinal tract, right superior longitudinal fasciculus, and right inferior fronto-occipital fasciculus with highest to lowest probability respectively. They, along with MO $(t>3$, $\left.\mathrm{FWE}_{\text {corrected }} \mathrm{P}<0.05\right)$, were decreased in right inferior front-occipital fasciculus, inferior longitudinal fasciculus, and anterior thalamic radiation. They were also commonly affected in cingulum and superior longitudinal fasciculus. Similarly, we also labeled the DTI data based on the JHU ICBM-DTI-81 WM Labels, FA and AD showed largest probability of decrease in right superior corona radiata, posterior corona radiata, posterior limb and retrolenticular part of internal capsule, posterior thalamic radiation, superior longitudinal fasciculus, and body of corpus callosum. AD also showed decrease in right cerebral peduncle. Although there was only a small probability of significant voxels of $\mathrm{FA}$ and $\mathrm{AD}$ in right external capsule, MO had the largest probability. MO was also decreased in right uncinate fasciculus.

The above findings are tabulated in Table 2 and illustrated in figure 1.

Table 1: Specific anatomic white matter regions showing significant voxels.

\begin{tabular}{|c|c|c|c|c|c|c|c|}
\hline & Size & $\mathbf{P}$ & $\mathbf{X}$ & $\mathbf{Y}$ & $\mathbf{Z}$ & JHU ICBM-DTI-81 White-Matter Labels & $\%$ Prob \\
\hline FA & 2988 & 0.05 & 37 & -48 & 5 & Unclassified & 41.33 \\
\hline \multicolumn{6}{|c|}{$\left(\mathrm{t}>1.5, \mathrm{FWE}_{\text {corrected }} \mathrm{P}<0.05\right)$} & Right Superior corona radiata & 18.74 \\
\hline & & & & & & Right Posterior corona radiata & 16.23 \\
\hline & & & & & & Right Posterior limb of internal capsule & 7.73 \\
\hline $\mathrm{AD}$ & 2408 & 0.044 & 16 & -15 & -12 & Right Superior corona radiata & 29.73 \\
\hline \multirow{3}{*}{\multicolumn{5}{|c|}{$\left(\mathrm{t}>1.5, \mathrm{FWE}_{\text {corrected }} \mathrm{P}<0.05\right)$}} & & Unclassified & 16.20 \\
\hline & & & & & & Right Posterior limb of internal capsule & 15.41 \\
\hline & & & & & & Body of corpus callosum & 13.21 \\
\hline MO & 75 & 0.024 & 37 & -7 & -18 & Unclassified & 50.67 \\
\hline \multicolumn{5}{|c|}{$\left(\mathrm{t}>3, \mathrm{FWE}_{\text {corrected }} \mathrm{P}<0.05\right)$} & & Right External capsule & 45.33 \\
\hline
\end{tabular}

$\mathrm{X}, \mathrm{Y}$, and $\mathrm{Z}$ co-ordinates are as per Montreal Neurological Institute standard in $\mathrm{mm}$ and represent the minimum $\mathrm{P}$ value. $\%_{\text {Prob }}=$ the probability of a voxel or mask being a member of the different labeled regions within the atlas. JHU $=$ John Hopkins University. 


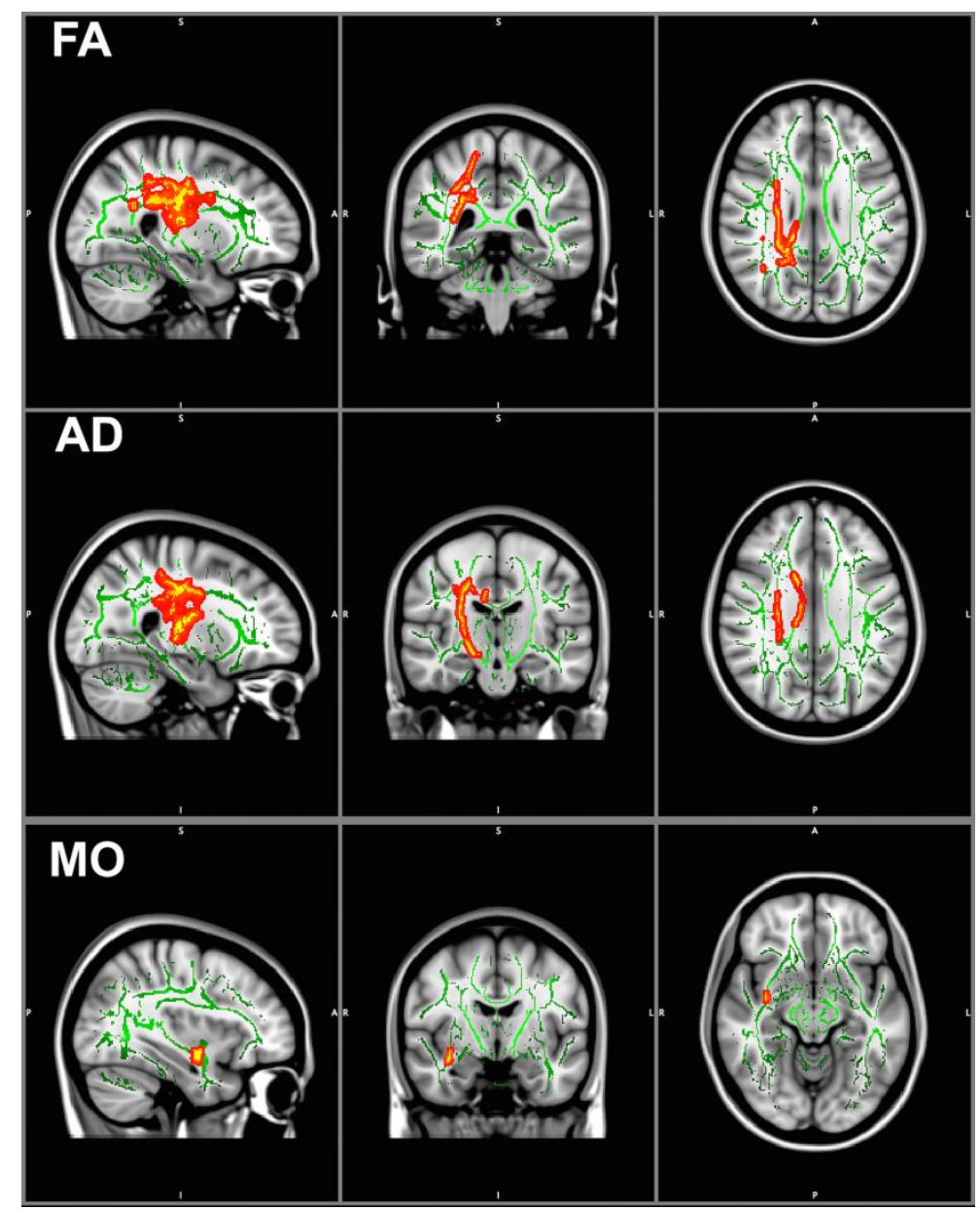

Figure 1: Decrease in FA, AD, and MO observed in Right sided tinnitus patients compared to controls after unpaired $\mathrm{T}$ test. The hot colors indicate changes in cluster size threshold $(\mathrm{t})>1.5$, $\mathrm{FWE}_{\text {corrected }} \mathrm{P}<0.05$ for FA and $\mathrm{AD}$, and $\mathrm{t}>3, \mathrm{FWE}_{\text {corrected }} \mathrm{P}<0.05$ for MO. The green lines indicate mean FA skeleton obtained from patients and controls. They are both painted over their respective anatomical location in the MNI standard brain image.

MO of the left-sided tinnitus patients, compared to controls, showed significantly decreased values $\left(t>1.5, \quad F W E_{\text {corrected }}\right.$ $\mathrm{P}<0.05)$ at Forceps major, bilateral cingulum (especially the left), and bilateral anterior thalamic radiation. Similarly, we labeled the DTI data based on the JHU

The above findings are illustrated in figure 2 .
ICBM-DTI-81 WM Labels. MO showed the largest probability of decrease in splenium of corpus callosum. Body of corpus callosum, left posterior corona radiate, and the left cingulum were also involved. 

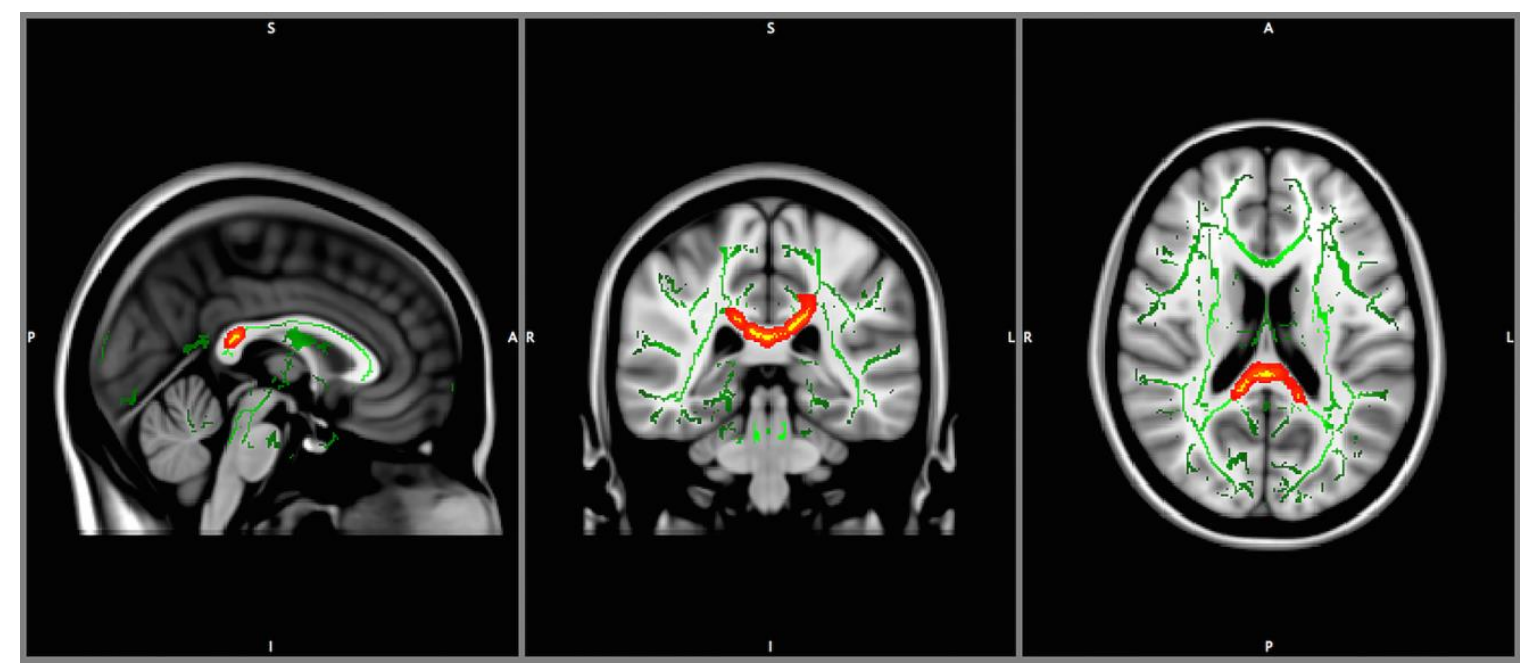

Figure 2: Decrease in $\mathrm{MO}$ observed in Left side tinnitus patients at $t>3, \mathrm{FWE}_{\text {corrected }} \mathrm{P}<0.05$. The hot colors indicate changes in $\mathrm{P}$ values $<0.05$. The green lines indicate mean FA skeleton. They are both painted over their respective anatomical location in the MNI standard brain image.

No significant clusters were observed after FWE correction in bilaterally affected patients in any DTI parameter.

\section{DISCUSSION}

The role of white matter integrity was successfully investigated. After analyzing the whole brain for white matter changes quantitatively using TBSS, changes were observed in unilaterally affected tinnitus patients compared to controls. In rightsided tinnitus patients, there were gross changes in all DTI parameters but significant clusters were observed in FA, $\mathrm{AD}$ and MO. Similarly, in the left sided tinnitus patients, gross changes were also observed, but significant clusters were only observed in MO. In bilaterally affected tinnitus patients, there were no gross or significant clusters. Tinnitus related neuronal dysfunction and plasticity are assumed to have caused white matter integrity changes and differed according to the affected side in our set of tinnitus subjects.
Tinnitus affects different modalities involving different networks. Resting-state functional connectivity studies take advantage of such state changing focus from a core region to a network, to study the pathophysiology of the disease. Therefore, multi-sensory association areas such as the cortico-cortical connections or sub-cortical connections through the thalamus probably play an important role supporting a model of thalamocortical dysrhythmia, in which the thalamus generates faulty signals resulting in altered cortical activity, could not be ruled out ${ }^{10}$. Thalamus is connected to the anterior cingulate gyrus through the anterior thalamic radiation, which corresponds to the findings of this study with decreased $\mathrm{FA}$ and $\mathrm{AD}$ values in right-sided tinnitus patients while MO in the left. The anterior cingulate gyrus has dorsal and sub-genual parts. The subgenual part, anti-correlated with the dorsal part, is functionally 
connected to amygdala, insula, parahippocampus, prefrontal, and orbitofrontal cortices forming a 'high route' of cortical connections ${ }^{11}$. Therefore, it is a key component of distress. Ipsilateral white matter around the (para)hippocampus were also involved in unilaterally affected patients. Parahippocampus is responsible for auditory memory and sensory gating of tinnitus, but was altered in our patients ${ }^{12}$. The anterior cingulate gyrus along with the insula forms a salience network, which is thought to cause improved sound detection thresholds ${ }^{13}$. Insula is also connected to the orbitofrontal cortex known to be responsible for autonomic and emotional responses ${ }^{14}$. It maintains its corticocortical connections to these regions via the external capsule, which was affected in unilaterally affected patients in this study.

The pyramidal tracts arise from pyramidal cells of cortical areas mostly the premotor cortex and are projected into the posterior limb of the internal capsule through the superior corona radiate and into the corticospinal tracts. Significantly decreased FA and $\mathrm{AD}$ values of these structures were found on the right side in the study, indicating the involvement of the non-classical pathway. The motor system is currently not considered to be directly involved with the pathophysiology of tinnitus, and such abnormality may reflect global changes ${ }^{15}$. However, primate studies show their relevance for tinnitus perception, and furthermore electroencephalographic studies have also revealed alterations in supplementary motor areas ${ }^{16}$.

Cross-modal plasticity could induce motor system changes due to sensory deprivation. Moller, A. R., et al. demonstrated distinct changes in tinnitus perception in 10 out of 26 of their patients ${ }^{17}$, and later showed that children also experienced changes in loudness perception $^{11}$. Therefore, the non-classical pathway was masked in adults, but unmasked in presence of tinnitus perception attributed to neural plasticity forming a 'low route'. Furthermore, tinnitus percept could be influenced by somatic maneuvers ${ }^{18}$ in some patients especially the temporomandibular joint ${ }^{19}$. Schecklmann, Landgrebe, Kleinjung, Frank, Rupprecht, Sand, Eichhammer, Hajak and Langguth $^{20}$ demonstrated treatments of repetitive transcranial magnetic stimulations of motor cortex induced its short-interval cortical inhibition change. Decrease in inhibition was associated with decrease in symptoms. They therefore speculated such alterations of motor cortex excitability, regulated by inhibitory $\gamma$-aminobutyric acid (GABA) and excitatory N-methyl-d-aspartate $\mathrm{NMDA}^{21}$, could be a consequence, a precondition of tinnitus, or both. Therefore, somatosensory network could be an important connection to explain the pathophysiology of tinnitus than previously thought.

The most common example of such crossmodal integration of network due to neural plasticity is audio-visual network. Auditory stimulation activates visual areas in animal studies 22,23 , and in human studies of blind subjects ${ }^{24,25}$. Moreover, Weeks, Horwitz, Aziz-Sultan, Tian, Wessinger, Cohen, Hallett and Rauschecker ${ }^{26}$ demonstrated in their PET study that auditory motion caused activation of visual cortical areas more on the right hemisphere. The right-sided 
tinnitus patients in this study had altered parameters in ipsilateral retro-lenticular part of internal capsule and optic radiations. These fibers originate from the lateral geniculate body of the thalamus.

Pathways, connecting posterior cingulate cortex posterior network to the thalamus via posterior corona radiata, were affected along with white matter changes around hippocampus. The anterior limb of the internal capsule, which connects the thalamus to the prefrontal cortex, was also affected in right-sided tinnitus patients. Together, they all form the subcomponents of the default network system, which appeared to be compromised in our set of patients ${ }^{27}$. Alterations in this network are known to cause insomnia, and Cranial Electrotherapy Stimulation is used to treat it by cortical deactivation causing connectivity changes to the network ${ }^{28}$. However, a DTI study of a patient with familial insomnia had alteration in thalamus and cingulate cortex ${ }^{29}$. Either of the possible mechanisms could explain the presence of insomnia in tinnitus patients.

Integrity of the right SLF is correlated to performance of orienting due to its connection between parietal and frontal lobes, and maintains the attention network with dominance of the right hemisphere in the majority ${ }^{30}$. The anterior cingulate gyrus and the prefrontal cortex via the anterior corona radiata control maintain executive control of attention, and studies have shown to have negative correlation with the $\mathrm{FA}$ values with $\mathrm{EC}^{31}$. IFOF connects the frontal and the temporaloccipital cortex, which probably maintains executive control as well ${ }^{32}$. All of these aforementioned white matter tracts were affected in the right-sided tinnitus patients suggesting altered attention state of orienting and executive control.

Thalamocortical dysrhythmia model, based on deafferentation, aimed to explain symptoms of movement disorders, epilepsy, neuropathic pain, chronic pain, and tinnitus ${ }^{33}$. Our results support this model with white matter changes centered around and in the thalamus, affecting multiple systems. The cross modal plasticity in somatosensory, auditory, and visual networks creates interacting network may have caused 'Synesthesia',34, in which stimulation of one modality can result in stimulation of cortices in another modality ${ }^{35}$. This could explain symptoms of gaze-evoked tinnitus and other forms of tinnitus evoking maneuvers in some patients ${ }^{35}$. Similarly, such plasticity even unmasks 'low routes' from the thalamus to the limbic system bypassing corticocortical connections of the "high route'"1, which may explain hyperacusis and further phonophobia experienced by some patients.

The right-sided tinnitus patients showed significant changes more towards the right hemisphere, while left-sided tinnitus patients showed more towards the left. However, the left-sided tinnitus also showed right-sided changes in the anterior thalamic radiation and cingulum of the anterior cingulate gyrus, when MOs were compared. The lack of results in other parameters could be due to a small sample size compared to right-sided tinnitus patients. However, bilaterally affected patients did not show any significant clusters even with relatively larger sample size. Further study is needed to draw inferences based on such negative findings. 
The modest number of patients limits the study, especially the left sided tinnitus patients. This could explain why rightsided tinnitus patients had more robust findings compared to little findings of leftsided tinnitus patients. However, in order to compensate for the age-related differences between subjects, we prepared an age-matched control to each of our subject. The patients also experienced some amount of hearing decline, which we have not taken into account. Therefore, hearing loss rather than tinnitus may have influenced the results, but Husain, Medina, Davis, Szymko-Bennett, Simonyan, Pajor and Horwitz ${ }^{7}$ in their DTI showed no effect. We have chosen to use a cluster extent threshold method for FWE correction rather than threshold free cluster enhancement (TFCE) because TFCE was more conservative in nature for the subset of data. Therefore, the selected threshold levels were completely arbitrary, which is a known methodological limitation.

\section{CONCLUSION}

Our observations show that white matter changes do occur in tinnitusattributed to its pathophysiological changes in unilaterally affected tinnitus patients compared to controls, but not in bilaterally affected patients. In these patients, there were white matter integrity changes in tracts of limbic, somatosensory, audio-visual, default mode, and attention network. These implied interactions of the networks, which were better explained by cross modal plasticity explaining symptoms, such as hyperacusis, phonophobia, gazed evoked tinnitus. ROI based tractography with fMRI study would define connectivity of each network, and confirm the findings in greater detail. We further add to the evidence of thalamocortical dysrhythmia model based on our observations. However, we did not observe any significant changes in bilaterally affected patients.Further studies are needed in this group to draw inferences.

\section{REFERENCES}

1. Jastreboff PJ. Tinnitus retraining therapy. Progress in brain research 2007; 166:415-23.

2. Cima RF, Andersson G, Schmidt CJ, Henry JA. Cognitive-behavioral treatments for tinnitus: a review of the literature. Journal of the American Academy of Audiology 2014;25(1):29-61.

3. Juris L, Andersson G, Larsen HC, Ekselius L. Cognitive behaviour therapy for hyperacusis: A randomized controlled trial. Behaviour research and therapy 2014;54:30-7.

4. Yoo DS, Choi WY, Lee SY, et al. Quantitative analysis of white matter on DTI images of patients with tinnitus: preliminary report. Conference proceedings : Annual International Conference of the IEEE Engineering in Medicine and Biology Society IEEE Engineering in Medicine and Biology Society Conference 2006;1:1870-2.

5. 5. Lee YJ, Bae SJ, Lee $\mathrm{SH}$, et al. Evaluation of white matter structures in patients with tinnitus using diffusion tensor imaging. Journal of clinical neuroscience: official journal of the Neurosurgical Society of Australasia 2007;14(6):515-9.

6. 6. Crippa A, Lanting CP, van DijkP, Roerdink JB. A diffusion tensor imaging study on the auditory system 
and tinnitus. The open neuroimaging journal 2010;4:16-25.

7. Husain FT, Medina RE, Davis CW, et al. Neuroanatomical changes due to hearing loss and chronic tinnitus: a combined VBM and DTI study. Brain research 2011;1369:74-88.

8. Smith SM. Fast robust automated brain extraction. Human brain mapping 2002;17(3):143-55.

9. Behrens TE, Woolrich MW, Jenkinson $\mathrm{M}$, et al. Characterization and propagation of uncertainty in diffusion-weighted MR imaging. Magnetic resonance in medicine: official journal of the Society of Magnetic Resonance in Medicine / Society of Magnetic Resonance in Medicine 2003;50(5):1077-88.

10. De Ridder D, van der Loo E, Vanneste $S$, et al. Theta-gamma dysrhythmia and auditory phantom perception. Journal of neurosurgery 2011;114(4):912-21.

11. Moller AR, Rollins PR. The nonclassical auditory pathways are involved in hearing in children but not in adults. Neuroscience letters 2002;319(1):41-4.

12. Grasby PM, Frith CD, Friston KJ, Bench C, FrackowiakRS, Dolan RJ. Functional mapping of brain areas implicated in auditory--verbal memory function. Brain: a journal of neurology 1993;116 ( Pt 1):1-20.

13. Seeley WW, Menon V, Schatzberg $\mathrm{AF}$, et al. Dissociable intrinsic connectivity networks for salience processing and executive control. The Journal of neuroscience: the official journal of the Society for Neuroscience 2007;27(9):2349-56.

14. Craig AD. Interoception: the sense of the physiological condition of the body. Current opinion in neurobiology 2003;13(4):500-5.

15. De Ridder D, Elgoyhen AB, RomoR, Langguth B. Phantom percepts: tinnitus and pain as persisting aversive memory networks. Proceedings of the National Academy of Sciences of the United States of America 2011;108(20):8075-80.

16. Lemus L, Hernandez A, Romo R. Neural encoding of auditory discrimination in ventral premotor cortex. Proceedings of the National Academy of Sciences of the United States of America 2009;106(34):14640-5.

17. Moller AR, Moller MB, Yokota M. Some forms of tinnitus may involve the extralemniscal auditory pathway. The Laryngoscope 1992;102(10):1165-71.

18. Sanchez TG, Rocha CB. Diagnosis and management of somatosensory tinnitus: review article. Clinics 2011;66(6):1089-94.

19. Vielsmeier V, Strutz J, Kleinjung T, et al. Temporomandibular joint disorder complaints in tinnitus: further hints for a putative tinnitus subtype. PloS one 2012;7(6):e38887.

20. Schecklmann M, Landgrebe M, Kleinjung $\mathrm{T}$, et al. State- and traitrelated alterations of motor cortex excitability in tinnitus patients. PloS one 2014;9(1):e85015.

21. Salvi R, LobarinasE, Sun W. Pharmacological Treatments for Tinnitus: New and Old. Drugs of the future 2009;34(5):381-400.

22. Kahn DM, Krubitzer L. Massive cross-modal cortical plasticity and the emergence of a new cortical area in developmentally blind mammals. Proceedings of the National Academy of Sciences of the United States of America 2002;99(17):11429-34.

23. Heil P, Bronchti G, WollbergZ, Scheich H. Invasion of visual cortex by the auditory system in the naturally blind mole rat. Neuroreport 1991;2(12):735-8. 
24. Kujala T, Huotilainen M, Sinkkonen J, et al. Visual cortex activation in blind humans during sound discrimination. Neuroscience letters 1995;183(12):143-6.

25. Cohen LG, Celnik P, Pascual-Leone A, et al. Functional relevance of crossmodal plasticity in blind humans. Nature 1997;389(6647):180-3.

26. Weeks R, Horwitz B, Aziz-Sultan A, et al. A positron emission tomographic study of auditory localization in the congenitally blind. The Journal of neuroscience: the official journal of the Society for Neuroscience 2000;20(7):2664-72.

27. Raichle ME, MacLeod AM, Snyder AZ, Powers WJ, GusnardDA, Shulman GL. A default mode of brain function. Proceedings of the National Academy of Sciences of the United States of America 2001;98(2):676-82.

28. Feusner JD, Madsen S, Moody TD, et al. Effects of cranial electrotherapy stimulation on resting state brain activity. Brain and behavior 2012;2(3):211-20.

29. Gemignani A, Laurino M, Provini F, et al. Thalamic contribution to Sleep Slow Oscillation features in humans: a single case cross sectional EEG study in Fatal Familial Insomnia. Sleep medicine 2012;13(7):946-52.

30. HeilmanKM, Van Den Abell T. Right hemisphere dominance for attention: the mechanism underlying hemispheric asymmetries of inattention (neglect). Neurology 1980;30(3):327-30.

31. Wakana S, Jiang H, Nagae Poetscher LM, van Zijl PC, Mori S. Fiber tractbased atlas of human white matter anatomy. Radiology 2004;230(1):7787.

32. Ge $\mathrm{H}, \mathrm{Yin} \mathrm{X}, \mathrm{Xu} \mathrm{J}$, et al. Fiber pathways of attention subnetworks revealed with tract-based spatial statistics (TBSS) and probabilistic tractography. PloS one 2013;8(11):e78831.

33. Llinas RR, Ribary U, Jeanmonod D, KronbergE, Mitra PP. Thalamocortical dysrhythmia: A neurological and neuropsychiatric syndrome characterized by magnetoencephalography.

Proceedings of the National Academy of Sciences of the United States of America 1999;96(26):15222-7.

34. Jacobs L, Karpik A, BozianD, Gothgen S. Auditory-visual synesthesia: sound-induced photisms. Archives of neurology 1981;38(4):211-6.

35. Cacace AT. Expanding the biological basis of tinnitus: crossmodal origins and the role of neuroplasticity. Hearing research 2003;175(1-2):11232. 